Z Herz-Thorax- Gefäßchir 2008 · 22:81-90 DOI 10.1007/s00398-008-0624-6 (C) Springer Medizin Verlag 2008

\author{
A. Albert · J. Ennker \\ Klinik für Herz-Thorax- und Gefäßchirurgie, Herzzentrum Lahr/Baden, BRD
}

\section{Sukzessive Beschreibung einer chirurgischen Technik}

\title{
OPCAB und komplette arterielle Revaskulari- sation als Methoden erster Wahl
}

plus Video

Zusätzliches elektronisches Material ist zu diesem Beitrag verfügbar unter DOI 10.1007/s00398-008-0624-6

\section{Einleitung}

Bypassoperationen werden heutzutage von einigen Teams standardmäßig auch ohne die Unterstützung der Herz-Lungen-Maschine (HLM) durchgeführt. OP$\mathrm{CAB}$ (off-pump coronary artery bypass) ist (nur) in Händen erfahrener Teams sowohl sicher als auch effektiv und kann bei allen Patienten, von seltenen Ausnahmen wie Patienten im kardiogenen Schock ausgenommen, angewandt werden. Ziel von OPCAB ist es, mit der Anwendung der HLM einhergehende Risiken und Belastungen für den Patienten vollständig zu vermeiden und dadurch die - ohnehin sehr niedrigen - Komplikationsraten der Bypassoperation noch weiter zu senken (siehe Abschnitt OPCAB-Ergebnisse). Um jedoch die Chancen dieser schonenden Operation tatsächlich zu nutzen, muss der Chirurg die OPCAB-Techniken mühelos beherrschen, allem voran die schonende Exposition und Stabilisierung des Herzens sowie die Naht der Anastomosen in engen Räumen und ungewohnten Winkeln. Insbesondere intraoperative Low-output-Phasen sowie unzureichende Anzahl und Qualität der Anastomosen sind nicht zulässig, da sie den Patienten außerordentlich gefährden können; die Operation soll jederzeit kontrolliert und ohne Stress für Chirurgen und Team ablaufen. Die Vergangenheit hat gezeigt, dass dies leider häufig nicht der Fall ist, weshalb wohl viele OPCAB-Programme in den Kliniken wieder eingestellt wurden. Die zum Teil widersprüchlichen Ergebnisse der OPCAB-Chirurgie, was beispielsweise die Offenheitsrate der Bypässe betrifft $[1,2]$, lassen sich mit statistisch-methodischen Unzulänglichkeiten mancher Studien, sowie mit unzureichend standardisierten Techniken und mangelnden OPCAB Erfahrungen der beteiligten Chirurgen erklären [3]; beides Probleme z.B. der bekannten Studie von Khan et al. [1]. Der Chirurg, welcher Bypassoperationen ohne HLM durchführen möchte, sollte auf eine sichere, reproduzierbare Technik zurückgreifen und bereit sein, für deren Anwendung intensiv zu trainieren (siehe Abschnitt OPCAB-Training).

\section{OPCAB-Techniken}

Die hier vorgestellte Technik sowie die Art und Weise, sie zu lehren, entspringt der auf CMEST(continuing medical education of surgical technologies)-Kriterien basierenden OPCAB-Schule von P. Sergeant in Leuven/Belgien. Sie ist sowohl im Internet (http://www.opcab-training.eu) als auch auf der den Trainingskurs begleitenden CD-ROM und im Lehrbuch [4] beschrieben. Der Trainingskurs mit all seinen vielfältigen Aspekten findet sich auch unter [5] erläutert. Die Methode hat sich unter verschiedensten Bedingungen als effektiv und erlernbar erwiesen. Wir beschreiben hier eine modifizierte Form (z.B. Verwendung skeletierter IMA, abweichende Reihenfolge der Anastomosen, Verwendung eines Sprühers), die wir auf nahezu das gesamte Spektrum unserer Bypasspatienten anwenden. Seit 2004 werden nach diesem Standard in unserer Klinik ca. 450 Operationen pro Jahr von mittlerweile 15 Chirurgen, darunter mehreren Ausbildungsassistenten, durchgeführt. 


\section{Lernmethoden}

Das Szenario, bei dem in der Hand des Unerfahrenen die OPCAB-Operationen unter instabiler Hämodynamik, entsprechendem Zeitdruck und auf hohem Stressniveau für den Chirurgen, aber auch das gesamte Team und letztlich den Patienten abläuft, ist bekannt. Mit wachsender Erfahrung und einer genügend großen Anzahl operierter Patienten wird sich der Operationsablauf verbessern; aus dem Kampf wird allmählich eine Kunst. Diese Art zu lernen (fire-fighting, artisan skills) basiert maßgeblich auf operativem Lernen (learning-by-doing) und ist als alleinige Methode nicht zu empfehlen [6]: einerseits, weil sie mit einer die Patienten gefährdenden Lernkurve einhergeht, andererseits, weil sie der hohen, für Lernfortschritte jedoch benötigten Fallzahl wegen nur von wenigen Chirurgen in einer Klinik erlernt werden kann.

Vielmehr sollte das Erlernen von OP$\mathrm{CAB}$ in systematischer Form erfolgen und wenig Raum für Unsicherheiten lassen (konzeptuelles Lernen). Die OPCABTechnik muss folgende Kriterien erfüllen:

- anwendbar auf ein breites Spektrum der Bypasspatienten

- sicher durchzuführen mit einer geringen Anzahl von Konversionen

- optimale Anzahl und Qualität der Anastomosen

zudem sollte sie

- reproduzierbar und von vielen Chirurgen erlernbar sein

Das OPCAB-Training sollte bestimmten Regeln folgen [5]. Eine längere Begleitung durch Experten, interaktive Diskussionen und Trockenübungen in einer Trainingsbox sind außerordentlich zu empfehlen (siehe Abschnitte OPCAB-Training und OPCAB-Implementierung). Zudem hat es sich als hilfreich erwiesen, die Prozedur in kleinere Lerneinheiten zu gliedern. Dementsprechend ist der nachfolgende Beitrag aufgebaut:

1) OPCAB-Techniken werden schrittweise von der Auswahl des Bypassmaterials und der Anastomosentechnik (Schritt 1) bis zur Fertigstellung aller Bypässe (Schritt 8) dargestellt.
2) Wichtige Bestandteile unserer Technik sind fett hervorgehoben und mit einem Aufzählungszeichen gekennzeichnet $(*)$.

3) In den Fällen, in denen wir von der Leuven-Technik abwichen, wird dies mit Verweis auf dieselbe angezeigt $(>$ klassische Leuven-Technik) und kommentiert; dort, wo kein expliziter Hinweis zu finden ist, stimmt unsere Technik präzise mit der in Leuven eingeführten Technik überein.

4) Andere gebräuchliche Techniken, wie sie beispielsweise in Lehrbüchern Erwähnung finden $[7,8,9]$, werden hier lediglich angerissen und ebenfalls durch einen Hinweis ( $>$ andere Techniken) gekennzeichnet.

5) Mit einem Ausrufezeichen (!) werden Punkte angekündigt, die besonders beachtet werden sollten, da sie in der Vergangenheit zu Problemen führten.

In der Online-Version des Artikels wird begleitend ein Film präsentiert, der die hier beschriebenen Techniken darstellt.

\section{OPCAB-Technik der Anästhesie}

Das wichtigste Prinzip einer OPCAB-Anästhesie ist die Optimierung von Symphatikustonus, Sauerstoffangebot und myokardialer Kontraktion. Der Anästhesist ist während der Operation insbesondere von der Operationsweise des Chirurgen abhängig. Der Chirurg muss jede relevante Aktion (Berührungen und Veränderungen der Lage des Herzens, Unterbrechungen des Koronarflusses, Shunteinlage, Freigabe des Bypassflusses etc.) dem Anästhesisten mitteilen (team approach). Die Luxation des Herzens, die Stabilisierungsmaßnahmen sowie die Fertigung der Anastomosen müssen so sicher erfolgen, dass Störungen der Herzfunktion weder durch mechanische Beeinträchtigungen noch durch myokardiale Ischämie auftreten. Bei entsprechend sorgfältiger chirurgischer Technik kann die OPCABAnästhesie in der Mehrzahl der Fälle auch von jüngeren, weniger erfahrenen Anästhesisten durchgeführt werden. Ein Anästhesist mit OPCAB-Erfahrung sollte jedoch erreichbar sein. Andererseits können bei unsachgemäßer Chirurgie auch erfahrene Anästhesisten keine sichere Hämodynamik gewährleisten. Im Einzelnen werden folgende anästhesiologische Techniken angewandt und empfohlen:

* Epikardiale Vorhof- und Ventrikelschrittmacher vorlegen und testen. Erst bei hämodynamisch relevanter Bradykardie sollte stimuliert werden (!). Der Vorhofschrittmacher sollte vom rechten Ventrikel mindestens $2 \mathrm{~cm}$ entfernt liegen, da ansonsten während der Herzluxation statt des Vorhofs der Ventrikel stimuliert werden könnte (!). Da sie sonst bei den Hinterwandanastomosen hinderlich sein können, sollten die Schrittmacherkabel nach rechts ausgeleitet werden.

> Klassische Leuven-Technik: Hier werden die Schrittmacherkabel lediglich vorgelegt, wenn der Patient präoperativ ein auffälliges EKG (RSB, LSB, AV-Block I bis III) aufweist.

* Optimierung der Vorlast durch selektive Anhebung der Beine. Dies hilft, Volumen und Katecholamine zu sparen.

> Andere Techniken: Die Trendelenburg-Lagerung ist nicht zu empfehlen, da der Chirurg einerseits die Lageveränderung des Situs kompensieren muss (Stress!), andererseits sich der Rückfluss aus den Kopfgefäßen verschlechtern kann (Gefahr eines Hirnödems).

* Aufrechterhalten einer konstanten Körpertemperatur des Patienten durch Wärmedecke (bear hugger) in Ergänzung zu warmen Infusionslösungen, erwärmten Beatmungsgasen, angenehmer Raumtemperatur und warmer Gelmatratze.

> Klassische Leuven-Technik: Verzicht auf Wärmedecke oder andere spezielle Systeme zur Aufrechterhaltung der Temperatur; es wird allein eine warme Gelmatratze eingesetzt und die Raumtemperatur, bereits während der Einleitung, sorgfältig gesteuert.

* Volle Antikoagulation (Heparin 3 mg/ kgKG mit einem Zielwert von einer ACT > 400)

$>$ Andere Techniken: Einige Autoren verwenden eine reduzierte Antikoagulation mit niedrigeren Zielwerten. Dies könnte in Anbetracht der individuell sehr unterschiedlichen Ver- 
änderungen der Heparinkonzentration über die Zeit, der Thrombogenität intrakoronarer Shunts und des häufig schlechten Koronarstatus der Patienten das Risiko von Thrombenbildung und Bypassverschlüssen jedoch erhöhen.

* Vollständige Antagonisierung des Heparins

> Andere Techniken: Einige Autoren empfehlen, lediglich die Hälfte des Heparins zu antagonisieren, ggf. auf Grundlage einer direkten Bestimmung der Heparinkonzentration im Blut. Ob dies wie erwünscht die Offenheitsrate der Bypässe erhöht, oder, wie zu befürchten, mit einem vermehrten Blutverlust einhergeht, wurde bisher nicht untersucht.

* Cell-Saver wird stets aufgebaut. In Abhängigkeit von der Menge des gesammelten Bluts wird gewaschen.

* Swan-Ganz-Katheter für eine intermittierende Messung des HZV und eine kontinuierliche der Pulmonalisdrücke (!). Der diastolische Pulmonalisdruck hat sich neben der Inspektion des Myokards als der sensitivste und am schnellsten reagierende Indikator myokardialer Ischämien erwiesen.

$>$ Klassische Leuven-Technik: Immer TEE und kontinuierliche HZV-Messung.

> Andere Techniken: Um die Kosten des Swan-Ganz-Katheters zu sparen, kann man auch eine direkte Punktion der A. pulmonalis und Messung dort durchführen. Das HZV muss in diesem Falle jedoch geschätzt werden, beispielsweise visuell durch das Integral unter der arteriellen Blutdruckkurve.

* Keine primär inotropen Katecholamine, da sie den Sauerstoffbedarf des Myokards erhöhen und die Schwelle zu malignen Rythmusstörungen senken.

* Einsatz von Noradrenalin oder reinen a-Agonisten wie Epinephrin.

\section{Chirurgische OPCAB-Technik}

1. Welches Bypass-Graft und welche Anastomosentechnik wählen?

Die Inzidenz neurologischer Komplikationen nach Bypassoperationen hängt

Z Herz-Thorax- Gefäßchir 2008 $\cdot 22: 81-90$

DOI 10.1007/s00398-008-0624-6

(c) Springer Medizin Verlag 2008

\section{A. Albert · J. Ennker}

\section{OPCAB und komplette arterielle Revaskularisation als Methoden erster Wahl. Sukzessive Beschreibung einer chirurgischen Technik}

\section{Zusammenfassung}

OPCAB (off-pump coronary artery bypass) in Kombination mit einer kompletten arteriellen Revaskularisation (total arterial revascularization = TAR) und der Verzicht auf jegliche Manipulation an der Aorta bieten gute Chancen, die Ergebnisse der Bypassoperation zu optimieren. Um dies zu erreichen und gleichzeitig ungünstige Lernkurven zu vermeiden, muss der Chirurg zunächst auf eine Technik zurückgreifen können, welche reproduzierbar und auf (nahezu) alle Patienten anwendbar ist sowie zu optimaler Zahl und Qualität der Anastomosen führt: Entscheidungsalgorithmen zu Wahl und Anordnung der Grafts, Methoden der IMA-Präparation, Gefäßdarstellung, Stabilisierungsmaßnahmen und Shunteinlage sowie der Anastomosenfertigung werden hier schrittweise beschrie- ben (und in der Online-Version in einem Film demonstriert). OPCAB-Training nach CMEST (continuing medical education of surgical techniques)-Kriterien umfasst auch die Beschäftigung mit chirurgischer Philosophie und Motivation, anästhesiologischem Management, OP-Ausstattung und -Management sowie Ergebnisanalysen. Zur erfolgreichen Umsetzung des OPCAB-Programms wird die Begleitung durch einen Experten und die Schaffung günstiger Implementierungsbedingungen in den Kliniken empfohlen. Diese werden letztlich von den Fähigkeiten des OPCAB-Chirurgen und dem Management des Chefs geprägt.

\section{Schlüsselwörter}

Bypassoperation - OPCAB - totale arterielle Revaskularisation · CMEST · Training

\section{Total arterial OPCAB as the method of choice: Stepwise description of the surgical technique}

\section{Abstract}

The combination of OPCAB with total arterial revascularization and aortic-no-touch technique offers the chance to further improve the results of CABG. In order to avoid unfavorable learning curves, the surgeon must use only standardized OPCAB techniques, which 1) can be applied safely to the whole spectrum of CABG patients, and 2) offer the same number and quality of anastomoses. Here we describe such a technique step-by-step starting with a decisionmaking algorithm for selection and arrangement of the grafts, the methods of IMA harvesting, visualization and stabilization of the coronary vessels, shunt insertion, ending with completion of the anastomo- ses (demonstrated by a film added in the online version of the manuscript). OPCAB training according to the principles of CMEST also deals with surgical philosophy, anesthesia techniques, management of the operating theater and analysis of OPCAB results. The successful implementation of OPCAB is afforded by supervision of an OPCAB expert. Finally, the manual and cognitive skills of the OPCAB surgeon and the management capability of the head surgeon determine the outcome of the OPCAB implementation process.

\section{Keywords}

CABG - OPCAB · CMEST · training · total arterial revascularization 
vom Ausmaß der Manipulationen an der Aorta ab [12, 13, 3]. Die vollständige Vermeidung jeglicher Manipulation an der Aorta kann erreicht werden, wenn OPCAB und T-Graft-Technik kombiniert werden. Mehr als $90 \%$ der Bypassoperationen können so durchgeführt werden, dass ein RIMA-Sequenzialgraft zu den Seiten- und Hinterwandgefäßen T-förmig in die LIMA inseriert wird (• Abb. 1). Anstelle der RIMA kann auch eine Vene (- Abb. 2) oder eine Radialarterie verwendet werden (• Tabelle 1$)$.

2. Welche Länge benötigt der SequenzialGraft? Ist die RIMA lang genug?

Die Länge, welche ein Bypass-Graft benötigt, um von seiner Insertionsstelle in der LIMA über die Anastomosen zu den Seiten- und Hinterwandgefäßen bis hin zum RIVP zu gelangen, hängt von der Zirkumferenz des linken Ventrikels ab. Es ist hilfreich, bereits im Vorfeld der Operation und spätestens während der MammariaPräparation die erforderliche Länge des Bypass-Graft zu kennen. Wir haben in einer bisher unveröffentlichen Studie die erforderlichen Längen bei mehr als 100 Patienten während der OPCAB-Operation gemessen und sind zu dem Fazit gelangt, dass man die erforderliche Länge mittels einer simplen Formel abschätzen kann:

* Geschätzter RIVA-RIVP-Abstand = [enddiastolischer Durchmesser (EDD) + Durchmesser der Sentum- oder Hinter-Wand $(\mathrm{DW}) \times 2] \times 3,14-(\mathrm{EDD}+$ $\mathrm{DW} \times 2)=(\mathrm{EDD}+\mathrm{DW} \times 2) \times 2,14 \cdot R e-$ chenbeispiel: Die erforderliche Länge des Bypass-Graft bei einem hypertrophierten und leicht dilatierten Herz $(\mathrm{EDD}=5,5 \mathrm{~cm}$ und einem $\mathrm{DW}=$ $1,4 \mathrm{~cm}$ ) errechnet sich folgendermaßen: $[5,5 \times(2 \times 1,4)] \times 2,14=17,8 \mathrm{~cm}$. Als leicht zu merkende Daumenregel kann ebenfalls gelten: $2 \times$ EDD $+4 \times$ $\mathrm{DW}+\mathbf{1}$. Unter der Voraussetzung, dass der Sequenzial-Graft wie in den $\bullet \mathbf{A b}$ bildungen 1-2 zu sehen ohne einen Bogen zu machen in gestreckter Form von einer Anastomose zur anderen gelegt wird, ergaben die Berechnungen nach dieser Formel durchschnittlich $0,5 \mathrm{~cm}$ mehr als die tatsächlich erforderliche Länge; unterschätzt wird die erforderliche Länge unserer Erfahrung nach nicht.

\begin{abstract}
Abb. 1 Darstellung einer RIMA, die als T-

Graft in die LIMA inseriert wurde und als Sequenzial-Graft 2 PLAs und den RIVP versorgt. Der HerzPositionierer ist auf der anterioren Wand angesaugt, unter Meidung des epikardialen Fetts am Übergang zur diaphragmalen Seite. Das Herz wird von unten durch die am Boden des Perikards verankerte Kompresse gestützt und entlastet.
\end{abstract}

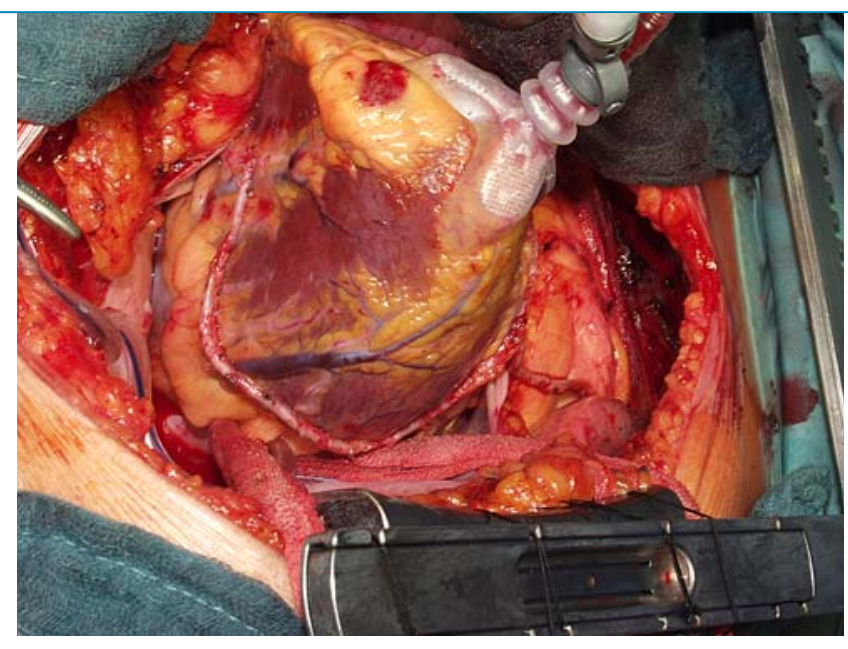

Abb. 2 Lage des Herz-Positionierer und der stets auf der linken Seite fixierten Kompressenschlinge wie in Abb. 1. Die Schrittmacherkabel sind rechts ausgeleitet, der Sperrer wird mit der Querschiene nach oben weisend eingesetzt. Hier wurde für die Anastomosierung eine $R D$, eines PLA und des RIVP eine Vene verwendet und als T-Graft in die LIMA inseriert.

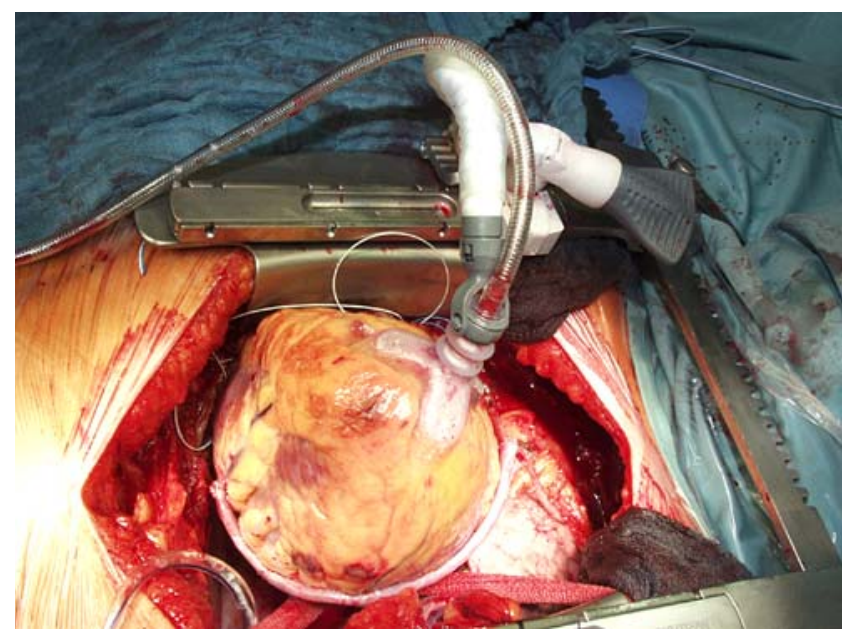

Tabelle 1

Entscheidungsalgorithmus zur Wahl der verschiedenen Bypasschirurgietechniken

$\begin{array}{ll}\begin{array}{l}\text { Entscheidungsfaktor } \\ \text { Methode erster Wahl! }\end{array} & \begin{array}{l}\text { Empfohlene Bypass-Technik } \\ \text { RIMA-Sequenzial-Graft wird als T-Graft in die } \\ \text { LIMA inseriert }\end{array} \\ \begin{array}{l}\text { hohes Risiko einer Mediastinitis* } \\ \begin{array}{l}\text { Notfalloperationen, ältere, multimorbide } \\ \text { Patienten }\end{array}\end{array} & \begin{array}{l}\text { Sequenzial-Graft mit einer Vene wird als } \\ \text { T-Graft in die LIMA inseriert }\end{array} \\ \begin{array}{l}\text { hohes Risiko einer Mediastinitis, keine } \\ \text { brauchbaren Venen }\end{array} & \begin{array}{l}\text { Sequenzial-Graft mit A. radialis wird als } \\ \text { T-Graft in die LIMA inseriert }\end{array} \\ \begin{array}{l}\text { ausgesprochen dominante RCA oder für } \\ \text { T-Graft unbrauchbare IMA }\end{array} & \begin{array}{l}\text { LIMA zu LAD und Einzelvenen mit proxi- } \\ \text { maler Anastomose in die Aorta [hier ist das } \\ \text { Heart-string-System von Boston Scientific } \\ \text { (früher Guidant) zu empfehlen] }\end{array} \\ \end{array}$

* insofern mehr als zwei der folgenden Risikofaktoren vorliegen: schwere COPD, insulinpflichtiger Diabetes mellitus, starkes Übergewicht

* Ein ausgesprochen unkomplizierte Orientierung über die Länge des gewonnenen Bypass-Graft gibt eine gefaltete (trockene) Kompresse, die normalerweise $20 \mathrm{~cm}$ lang ist: RIMA oder Radialarterie, die so lang sind wie eine solche Kompresse, reichen stets aus. 
* Es ist nicht notwendig, Bögen zwischen den Anastomosen zu machen; die Seit-zu-Seit-Anastomosen werden in einem exakten $90^{\circ}$-Winkel angelegt (- Abb. 1, 2). Grundsätzlich hat man bei einer OPCAB-Operation den Vorteil, dass man die Anordnung des Sequenzial-Graft am normal gefüllten und kontraktilen Herzen ausmessen kann und diese nicht am erschlafften Herzen geschätzt werden muss. Daher kann das Graft-Material bis auf weniger als $1 \mathrm{~cm}$ genau genutzt werden.

3. Präparation der IMA und Fertigung der T-Graft-Anastomose

* Zur Präparation einer skeletierten IMA wird diese aus dem Bett zwischen den Begleitvenen herauspräpariert. Seitenäste werden entweder geklippt oder mit niedriger Leistung (Koagulationsstrom 20-30 W) versiegelt. Eine skeletierte Präparation hat den Vorteil einer klaren Sicht auf die gesamte IMA und deren Seitenäste, zudem geht sie mit einem geringeren Trauma für die sternale Mikrozirkulation und einer etwas größeren Länge einher ( $\bullet$ Abb. 3 ).

$>$ Klassische Leuven-Technik: Hier wird die IMA mit einem kleinen Pedikel entnommen (Koagulationsleistung $20 \mathrm{~W}$ ); wahrscheinlich ist die Gefahr von Verletzungen oder Knicks der IMA geringer als bei der skeletierten Technik. Auch die Länge ist ausreichend, der Nachteil besteht jedoch in der geringeren Übersichtlichkeit.

4. Sternotomie und Einschneiden des Perikards

* Es empfiehlt sich, den OPCAB-Sperrer so einzusetzen, dass die Querschiene nach kranial zeigt, da diese sonst bei Rechtshändern die Fertigung der Hinterwandanastomosennähte behindern kann (• Abb. 2).

* Das Perikard sollte weit nach links bis zum Apex eröffnet werden, um die Luxation des Herzens zu erleichtern.

* Fertigung der proximalen T-GraftAnastomose vor den distalen Anastomosen (• Abb. 4): dies birgt den Vorteil, dass in dem Moment, in welchem die distale Anastomose fertiggestellt wurde, das Koronargefäß über den Bypass sogleich mit Blut versorgt ist.

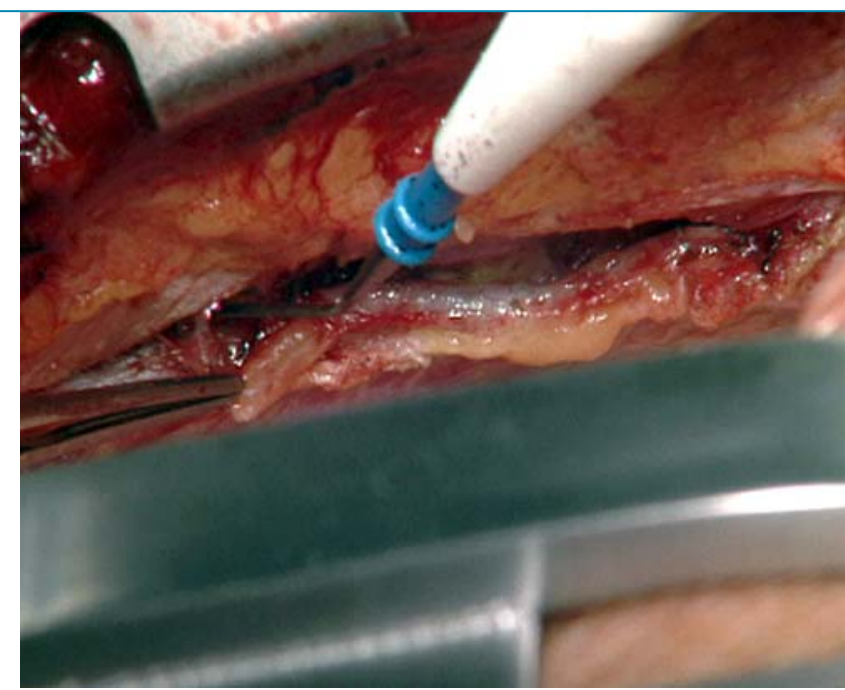

Abb. $3<$ Die LIMA wird aus ihrem Bett zwischen den Begleitvenen herausgeschält, die Seitenäste werden selten geklippt, sondern mit niedriger Koagulationsstromstärke (20-30 A) verschweißt.

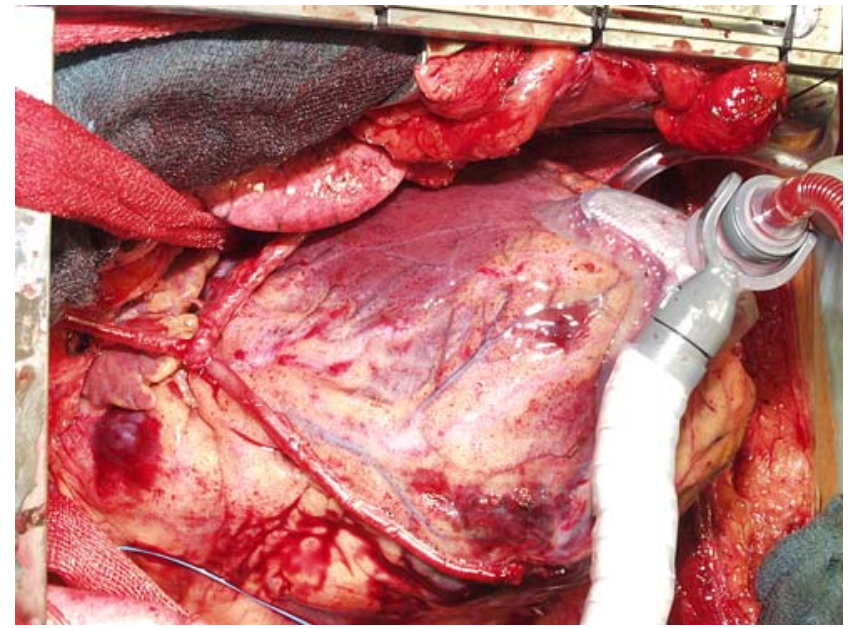

Abb. $4<$ Fertigung der T-Graft-Anastomose oben auf der LIMA und nicht an der Seite, um Knicke am Hals des Sequenzial-Graft zu vermeiden.

* Erfahrungsgemäß sollte die T-GraftAnastomose in die LIMA auf Höhe der Pulmonalklappe inseriert werden.

* (!) Wichtig ist es, die RIMA (Vene oder Radialarterie) stets oben auf die LIMA und nicht an die Seite zu setzen, weil der inserierende Graft dann am Hals abknicken könnte.

* (!) Wenn der Sequenzial-Graft nach der T-Graft-Anastomose zunächst mit einem Diagonalast anastomosiert werden soll, müssen der Ort der T-GraftAnastomose sowie Geometrie und Länge des inserierenden Graft sorgfältig bedacht werden. Manchmal ist es besser, eine Seit-zu-Seit-Anastomose mit der LIMA (LIMA-SequenzialGraft) durchzuführen, anstatt mit der RIMA einen Bogen zu machen, der Länge kosten und den Bypassfluss gefährden kann. Alternativ kann für diesen Diagonalast ein getrennter Bypass mit einem Reststück LIMA oder RI-
MA als zweite T-Graft-Anastomose in die LIMA gesetzt werden.

> Klassische Leuven-Technik: Hier wird die proximale Anastomose erst nach Ende der Fertigung aller distalen Grafts durchgeführt, um eine optimale Anordnung der Bypässe $\mathrm{zu}$ erreichen. Falls es Ischämien beim Ausklemmen der LIMA für die proximale Anastomose gibt, wird ein Shunt in die LIMA eingelegt.

* RIVA wird stets zuerst anastomosiert. Für diese Anastomose muss das Herz lediglich minimal manipuliert werden. Da routinemäßig Shunts verwendet werden, sind ischämische Probleme, selbst wenn der RIVA das kollateralisierende Gefäß ist, selten.

$>$ Andere Techniken: Eine klassische OPCAB-Regel besagt, dass man zuerst die verschlossenen oder kollateralisierten Gefäße anastomosieren solle; erst dann folgt das kollaterali- 
sierende Gefäß, um ischämische Probleme zu vermeiden.

5. Anastomosen auf RIVA und RD

1) Darstellung des RIVA: Ohne das Herz zu berühren, werden horizontale Stiche am linkslateralen Perikard auf Höhe des RIVA gemacht und das Herz sukzessive durch Zug an diesen Nähten so weit linkslateral angehoben, bis der RIVA sichtbar und stabilisierbar wird. Die Nähte werden dann am Sperrer oder mit einer Klemme am Bauchtuch bzw. den Seitentüchern fixiert. In seltenen Fällen (ca. 1 von 15) mit einer deutlichen Linksverlagerung des Herzens, bei denen durch diese Methode der RIVA nicht in die gewünschte Position zu bringen ist, kann hier bereits die Luxation des Herzens (Schritt I mit Kompressenschlinge) erfolgen (s.u.).

2) Anbringen des Stabilisators (Sog: $250 \mathrm{mmHg}$ ): Der Stabilisator wird stets an derselben Stelle angebracht und zwar am rechten unteren Sperrerrand. Entsprechend der Anatomie werden die Saugarme nach oben gebogen und gespreizt und der Oberfläche des Herzens angepasst (!). Eine Ansaugung von Diagonalästen sollte vermieden werden, ggf. werden die Sauglöcher an diesen Stellen mit Knochenwachs verschlossen (!). Je sorgfältiger die Saugarme der Oberfläche des Herzens angepasst werden, desto geringer ist der notwendige Druck auf den Ventrikel und desto geringfügiger ist die durch den Stabilisator hervorgerufene lokale Akinesie der Herzwand.

3) Temporäre Okklusion des Gefäßes zur Shuntinsertion (mit 4/o-Prolene und feinem Snugger) (• Abb. 5) (!). Im Falle eines schlecht funktionierenden Shunt, welcher wegen koronarer Plaques, Schlängelungen des RIVA oder deutlichen Differenzen des proximalen und distalen Durchmessers des Koronargefäßes nicht ausreichend abdichtet, kann das Gefäß in Ausnahmefällen für die Zeit der Anastomosennaht okkludiert werden. In diesem Fall ist der Anästhesist der Gefahr ischämischer Komplikation wegen zu informieren.

4) Wahl des Anastomosenareals: Die Wahl des Anastomosenareals ist insbesonde-

\begin{abstract}
Abb. 5 Haltenähtchen werden der Übersicht halber neben der Koronararterie evertierend gestochen (erste Nadel: innen_ außen_außen_innen), das Gefäß wird mittels des proximal der Anastomose angebrachten Snugger erst okkludiert nachdem die Inzision erfolgte, um sicher zu sein, dass das richtige Lumen eröffnet wurde.
\end{abstract}

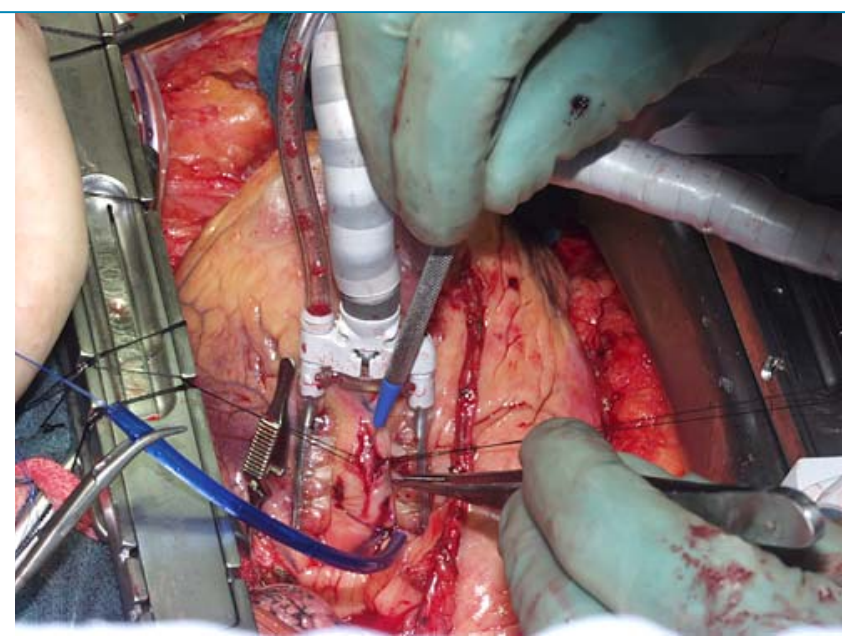

\title{
Abb.6 Erste Phase der Luxation des Her- zens mittels der Kom- pressenschlingen; man sieht, wie die Masse des linken Ventrikels zwischen den beiden Schenkeln der Kom- presse liegt, der RIVA zeigt in einem $90^{\circ}$-Win- kel nach oben. Das Herz hat eine sphärische Konfiguration ange- nommen, die Hämo- dynamik ist stabil (RR 140/60 mmHg, Pul- monalisdruck 34/12 $\mathrm{mmHg}, \mathrm{HZV}$ 5,2 l/min), es treten keine Rhyth- musstörungen auf.
}

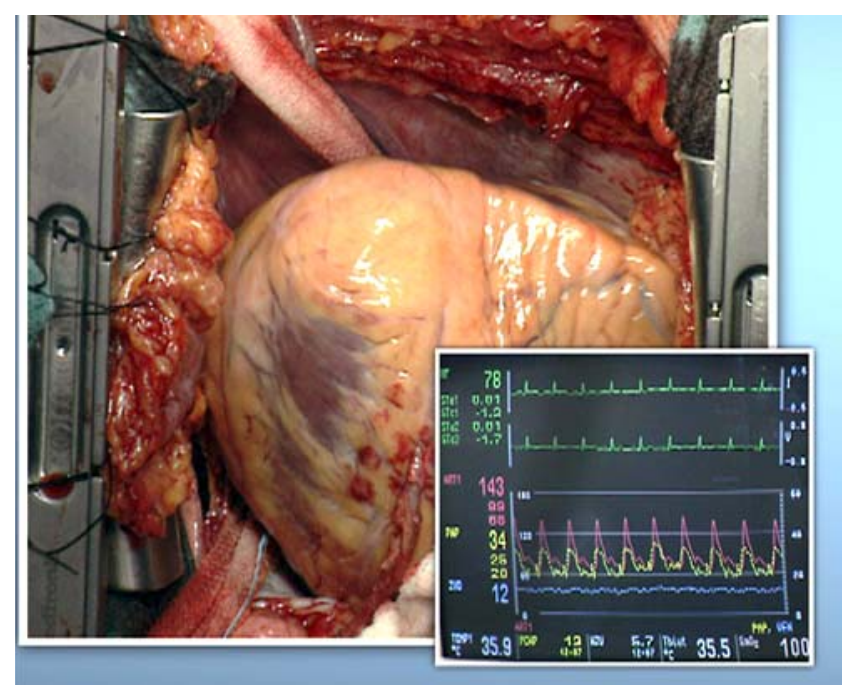

re beim RIVA von großer Bedeutung. Gefährliche Ischämien durch schlecht funktionierende Shunts treten, erfahrungsgemäß, wenn überhaupt, hier bei der RIVA-Anastomose auf. Daher sollte man, sofern möglich, eine Stelle mit einem Abstand von mindestens $1 \mathrm{~cm}$ von der nächsten größeren Plaque auswählen. Im Falle eines tief im epikardialen Fett oder intraseptal laufenden RIVA wird man diesen mittels eines Fettspreizers und evertierend gestochenen Haltenähten schrittweise freilegen.

5) Shuntinsertion und Anfertigung der Anastomose: es ist empfehlenswert beides, die Shuntinsertion und die Anastomosennaht in einer Trainingsbox zu üben. Dies gilt auch für Chirurgen, die bereits Erfahrung mit Bypassoperationen an der HLM haben (siehe Abschnitt OPCAB-Training und OPCAB-Implementierung). Die Shuntinsertion sollte so erfolgen, dass zuerst der längere Teil des Shunt proximal inseriert wird, und dann der kürzere unter einer Biegung (die vergleichbar ist mit dem Eintauchen eines GänseHalses in das Wasser) distal vorgeschoben wird. In besonderen Fällen, wenn beispielsweise nahe proximal der Anastomose ein großer Diagonalast abgeht, kann das längere Ende auch nach distal inseriert werden.

* Stets Einlegen eines Shunt: Damit gehen einher: weniger Ischämien, größere Sicherheit für den Patienten, weniger Stress für den Chirurgen, größere Sicherheit für die Anastomosennaht.

$>$ Andere Techniken: Shunts können Studien am Tiermodell zufolge Verletzungen des Endothels hervorrufen. Daher empfehlen einige Autoren die Shuntinsertion nur in Ausnahmefällen durchzuführen, z. B. wenn es während der Okklusion des Gefäßes zu Ischämien kommt. 
* Verwendung eines Sprühers: Diese hat sich doch als hilfreich erwiesen, insbesondere wenn Shunts nicht gut abdichten (!).

$>$ Klassische Leuven-Technik: Wegen des Risikos einer Verletzungsgefahr des Endothels an den IMA und des Auftretens von Luftembolien wird hier kein Sprüher verwendet, stattdessen eine Kompresse für den Chirurgen und eine Wasserspritze für den Assistenten.

* Evertierende Haltenähtchen: Diese Frage wird von den Chirurgen in unserer Klinik unterschiedlich gehandhabt, evertierende Haltenähtchen sind jedoch bei intramyokardial verlaufenden und von reichlich Fett umgebenen Koronargefäßen ausgesprochen hilfreich. Sie stabilisieren das Gefäß zusätzlich und sichern die Darstellung der lateralen Gefäßränder (• Abb. 5).

* 8/o-Prolene für arterielle Anastomosen (!): Insbesondere bei einer kleinkalibrigen LIMA ist die 8/o-Naht zu empfehlen. Wenn die IMA distal sehr eng ist, kann temporär ein Shunt oder eine Sonde eingelegt werden, um Einengungen im Halsbereich der LIMA zu verhindern.

* Beobachtung des Myokards (!): In regelmäßigen Abständen insbesondere nach Insertion des Shunt und Fertigstellung der Anastomosen sowie am Ende der Operation muss das Myokard hinsichtlich Farbe und Kontraktilität begutachtet werden. Zusammen mit dem diastolischen Pulmonalisdruck sind neu aufgetretene myokardiale Akinesien oder auch nur eine graublaue Farbe des Myokards die wichtigsten, sensitivsten und am schnellsten sichtbaren Indikatoren einer ernsthaften myokardialen Ischämie. In diesem Falle muss der Chirurg unverzüglich den Anästhesisten informieren und reagieren: Lagekorrektur des Herzens, evtl. Rücklagerung in das Perikard, Lösen aller Nähte, Korrektur der Shuntposition, Entfernen des Koronarstabilisators etc. (!). Falls die chirurgischen und anästhesiologischen Maßnahmen (Blutdruckkontrolle, antiischämische Therapie beispielsweise mit Nitroglyzerin) die Ischämie nicht sofort beheben können, muss eine Kon-

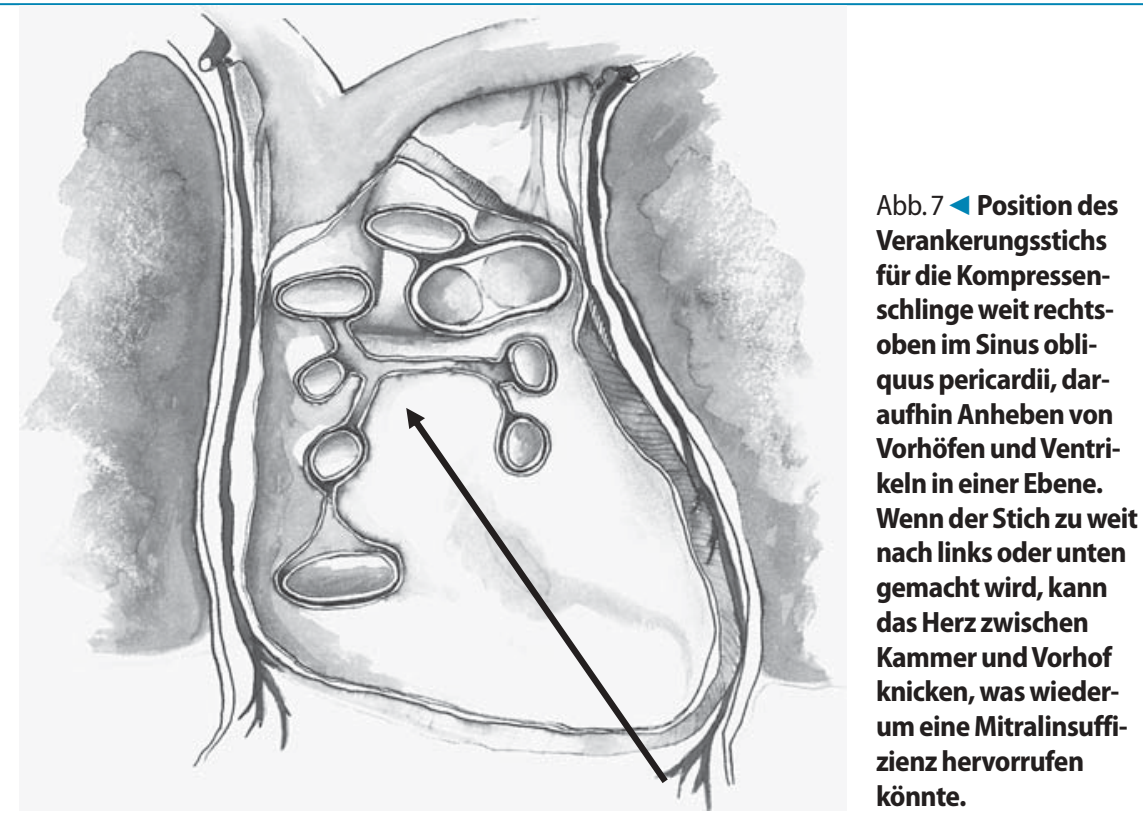

version zur HLM ohne Zeitverzug vorbereitet bzw. durchgeführt werden.

* (!) Mit dem Entschluss zur Konversion nicht zu lange warten! Je erfahrener das OPCAB-Team ist, desto seltener wird eine Konversion notwendig sein [10]. Die Konversionsrate der erfahrenen Chirurgen in unserem Haus beträgt ca. $1 \%$, in der Arbeitsgruppe in Leuven mittlerweile o, $1 \%$. Die Analyse jedes einzelnen Falles zeigte, dass hier vermeidbare chirurgische oder anästhesiologische Fehler gemacht wurden. Eine verspätete Konversion ist, so selten sie notwendig wird, das schwerste Vergehen bei OPCAB. Hier spielt die Kommunikation zwischen Chirurgie und Anästhesie eine enorm wichtige Rolle.

6. Luxation des Herzens I (mit Kompressenschlinge)

1) Vorbereitungen treffen zum Anbringen des Stichs zur Verankerung der Kompressenschlinge am Boden des Perikards (deep stitch)

a. 1/o-Prolene mit einem langen $\mathrm{Na}-$ delhalter bereitlegen

b. Korbsauger für den Assistenten bereithalten

c. trockene Kompressen, um das Herz anzuheben

2) Absprache mit der Anästhesie

3) Kurzes Anheben des Herzens (maximal 3-5s) mit der linken Hand (nachdem die rechte mit einer Kompresse den Weg für die linke Hand durch leichtes Anheben des Herzens freigemacht hat) bis die Sicht auf den gesamten Sinus obliquus frei wird. Anbringen der Naht im Sinus obliquus pericardii so hoch und rechts wie möglich (• Abb. 7) (!). Man beobachtet häufig, dass die linke Hand nicht tief genug unters Herz geht; die Fingerspitzen der linken Hand sollen bei dem Manöver unter dem Boden des linken Vorhofs liegen. Nur so ist der Sinus obliquus vollständig einsehbar.

4) Anbringen eines langen und steifen Tourniquets

5) Fixierung der Kompressenschlinge am Boden des Perikards und Platzierung der Masse des linken Ventrikels zwischen den beiden Armen der Schlinge (maximal 1-2 s)

6) Ankündigung der Luxation des Herzens für die Anästhesie

7) langsames Ziehen an beiden Schenkeln der Kompressen (!)

Die Masse des LV sollte nicht aus den Schenkeln herausrutschen, sonst ist eine Korrektur notwendig (• Abb. 6).

8) Anheben des Herzens bis der LAD $90^{\circ}$ nach oben zeigt

9) Sicherstellen, dass der rechte Ventrikel nicht gestaut ist

10) Optimierung der Vorlast a. selektives Anheben der Beine

b. Gabe von warmen Flüssigkeiten

c. Gabe von $\alpha$-Agonisten

(!) Durch diese Maßnahmen verändert sich die Geometrie des Herzens, 
es nimmt eine kugelige (sphärische) Form an (• Abb. 6). Bei Herzen mit normaler oder nur moderat gestörter Funktion ist der erste Schritt der Luxation möglich, ohne dass es zu bedeutenden Beeinträchtigungen der Hämodynamik kommt (CI > 2, RR systolisch $>80 \mathrm{mmHg}$ ). Bei deutlich vorgeschädigten Herzen, die mit einem stärkeren Druckabfall reagieren, sollte man sofort mit dem zweiten Schritt der Luxation fortfahren (Elongation, Apex-Stabilisierung), um eine günstigere (ellipsoide) Geometrie des Ventrikels zu erreichen.

7. Luxation des Herzens II (Elongation, Apex-Stabilisierung und Darstellung der posterolateralen Koronargefäße)

1. Rotation des Tischs nach rechts (!). Fixierung des rechten Beins

Die Rotation des Tischs nach rechts hat mehrere Vorteile: Zum einen wird der Druck der Schenkel der Kompresse vom Herzen genommen (!) (Ischämie durch Druck der Kompressenschlinge auf die CX), zum anderen wird der Raum für den Zugang zu den Seitenund Hinterwandgefäßen geöffnet.

2. Anbringen des Herz-Positionierers am rechten oberen Sperrerrand (Sog $200 \mathrm{mmHg}$, z. B. Starfish, Medtronic; Xpose, Boston Scientific). Die Saugglocke kann direkt auf den Apex oder auf der anterioren Wand angebracht werden. Beim Starfish werden 2 der 3 Arme parallel zum RIVA, um $1 \mathrm{~cm}$ nach links versetzt, angebracht. Saugen auf der diaphragmalen Seite oder dort, wo sich üppiges epikardiales Fett befindet, ist unsicher; der Starfish kann sich während der OP lösen. Nun wird das Herz etwas elongiert, so dass aus der kugeligen (sphärischen) eine ellipsoide Form entsteht. Dadurch wird das Herz auch von den Schenkeln der Kompressenschlinge abgehoben. Um das Herz weiter von unten zu unterstützen und das Eigengewicht von den Myokardfasern zu nehmen, werden die Kompressenschenkel nachgezogen.

3. Verlagerung des Herzens nach rechts, wodurch die Anastomosen zu den Seiten- und Hinterwandgefäßen möglich werden. Die Zugrichtung ist dabei schräg nach rechts oben, bis der rechte
Ventrikel gerade die rechte Sternumkante erreicht.

$>$ Andere Techniken: Einige Autoren empfehlen, die rechte Pleura zu inzidieren, um das Herz weiter nach rechts verlagern zu können. Diese extreme Rechtsverlagerung geht allerdings häufig mit schweren hämodynamischen Veränderungen einher und sollte daher nach unseren Erfahrungen nicht durchgeführt werden.

8. Anastomosen zu den Seiten- und Hinterwandgefäßen

RM und die PLA-Äste (evtl. RD) werden Seit-zu-Seit anastomosiert (DiamantAnastomosen) mit einem 90 -Winkel zwischen RIMA und Koronargefäß. Nachdem RIMA und Koronararterie mit jeweils zwei Stichen verbunden wurden (erste Nadel: RIMA: innen $\rightarrow$ außen $\rightarrow$ außen $\rightarrow$ innen; Koronargefäß: erste Nadel innen $\rightarrow$ außen, zweite Nadel: innen $\rightarrow$ außen) sollte die RIMA zum Koronargefäß runter gezogen werden, um den Rest der Anastomose dann vor Ort zu nähen. Das vermeidet Fadenverknotungen, deren Häufigkeit im engen Raum bei OPCAB größer ist, zum anderen hat der Assistent dann eine Hand frei, um beispielsweise den Faden zu führen. Um sich die Sicht in die Anastomose möglichst lange offen zu halten, sollte man stets am gegenüberliegendem und am schlechtesten einzusehenden Ende beginnen, also bei Diagonal- und hoch laufenden Marginalästen proximal am Bypass-Graft (rechts-lateral an der Koronararterie), bei PLA und RIVP lateral unten am Bypass-Graft (proximale Spitze an der Koronararterie).

\section{OPCAB-Training und -Implemen- tierung}

Eine Reihe von Möglichkeiten, sich über OPCAB zu informieren, sind geläufig: Informationsveranstaltungen bei Kongressen, Diskussionen, Video-Übertragungen von Live-Operationen, Lehrkurse auf CD-ROM, Trainieren am Tiermodell, ein bis zwei Tage dauernde Trainingskurse oder längere Trainingsaufenthalte (ein Monat bis ein Jahr) bei Experten, Einladen von Experten in der Klinik, Lehrbücher und Zeitschriften.
Vor- und Nachteile dieser Methoden kann man sich mittels einer Matrix veranschaulichen (www.ctsnet.org/sections/ newsandviews/inmyopinion Sergeant $P$, Wouters P. Introduction of a training matrix). Man erkennt relativ rasch, dass lediglich didaktisch gut aufbereitete Trainingskurse in einer Expertenklinik alle Lerninhalte für ein OPCAB-Training des Teams beinhalten, angefangen von der chirurgischen Philosophie und Motivation, dem anästhesiologischen Management, der Ausstattung des OP und dem Management im Saal, dem Erlernen der verschiedenen Techniken der Luxation, Stabilisierung, Shunteinlage, Anastomosennaht und der Bedeutung der Ergebnisanalyse. Wahrscheinlich sind längere Trainingsaufenthalte von mindestens drei bis sechs Monaten sinnvoll. Ein solcher Trainingsaufenthalt wird aber nur dann erfolgreich sein und in der Klinik, die ein Team zum Training geschickt hat, zur Implementierung von OPCAB mit guten Ergebnissen führen, wenn weitere Bedingungen erfüllt sind $[5,11]$. Die wichtigsten sind:

* Der Chirurg muss die OPCAB-Technik sicher beherrschen, dazu ist auch das Trainieren seiner manuellen Fähigkeiten an einem einfachen Modell (Trainingsbox) hilfreich (• Abb. 8).

* Der Chirurg sollte sich auf die Bypassoperation spezialisieren, zumindest so lange bis andere Chirurgen in OPCABVerfahren ausgebildet sind.

* Neben dem Chirurg muss ein Anästhesist ausgebildet und motiviert sein.

- Der Chef der Abteilung muss das OPCAB-Programm definieren und entsprechend durchsetzen. Es sollte Vorgaben existieren zu

$>$ Erwartungen und Zielen (angestrebter prozentualer OPCAB-Anteil in der Abteilung, Ergebnisanalysen, Patienteneinweisungswünsche) $>$ OPCAB-Strategie (Patientenselektion?)

$>$ Auswahl der OPCAB-Teams

Da ein Chef stets eine Vorbildfunktion inne hat, ist es günstig, er operiert selbst in OPCAB-Technik.

* Der ausgebildete OPCAB-Chirurg muss sein Wissen rasch den Kollegen vermitteln, wodurch das OPCAB-Programm bald auf mehreren Schultern ruht. 


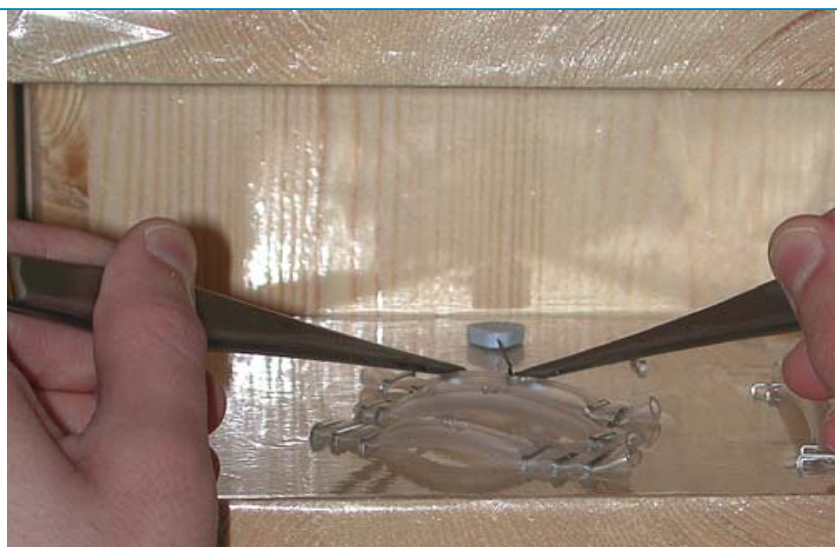

Abb. $8 \Delta$ Shuntinsertion und Anastomosennaht sollten zunächst außerhalb des Operationssaals trainiert werden; insbesondere die engen räumlichen Verhältnisse und ungewohnten Winkel bei OPCAB-Verfahren bedürfen des Trainings und können bereits in einer einfachen Trainingsbox, wie sie von der Arbeitsgruppe in Leuven verwendet wird, geübt werden.

\section{OPCAB-Ergebnisse}

Die International Society für Minimally Invasive Cardiothoracic Surgery (ISMICS) hat in Übereinstimmung auf der Grundlage von Metaanalysen mit Evidenzgraden A und B Aussagen zur OPCAB-Chirurgie formuliert [3], die sich in ihren wesentlichen Aussagen mit einer anderen Übersichtsarbeit decken [12]: Im Vergleich zur Bypassoperation mit HLM

- stellt OPCAB eine sichere Alternative bezüglich des Letalitätsrisikos dar [Klasse I, Level A]

- kann eine vergleichbare Vollständigkeit von Revaskularisation und Offenheitsrate der Bypässe erreicht werden, Voraussetzung sind der Einsatz eines modernen Equipments (Stabilisatoren, Herz-Positionierer) und ausreichende chirurgische Erfahrung [Klasse IIa, Level A]

- wird OPCAB empfohlen, um die perioperative Komplikationsrate zu senken [Klasse I, Level A]

- kann OPCAB empfohlen werden, um die Inzidenz postoperativer kognitiver Dysfunktionen zu senken [Klasse IIa, Level A]

- erreicht OPCAB eine gleichwertige Lebensqualität [Klasse I, Level A]

- wird OPCAB empfohlen, um Beatmungsdauer, Intensiv- und Krankenhausaufenthalt insgesamt zu senken und letztendlich Ressourcen zu sparen [Klasse I, Level A]

- sollte OPCAB insbesondere bei Hochrisikopatienten erwogen werden, um perioperative Letalität und Morbidität zu senken sowie Ressourcen zu sparen. [Klasse IIa, Level B]

Eine Arbeitsgruppe der American Heart Association (AHA) kommt in ihrer Metaanalyse hinsichtlich der Reduktion neurologischer Defizite zu ähnlich positiven Ergebnissen, stellt aber auch fest, dass die technischen Herausforderungen vielerorts nicht gemeistert wurden, was dann mit ausgeprägten Lernkuren, und einer geringeren Anzahl und Offenheitsrate der Bypässe bei den OPCAB-Operationen einherging [13]. Insofern kommt dem OPCAB-Training und dem Implementierungsprozess hier eine herausragende Bedeutung zu.

\section{Fazit für die Praxis}

Die Evidenzlage ermutigt, OPCAB einem breiten Patientenspektrum anzubieten. Der Chirurg muss sich jedoch der hohen technischen Herausforderungen des Verfahrens bewusst und auch wenn er bereits ein erfahrener (On-pump-)Bypasschirurg ist, bereit sein, spezielle OPCAB-Techniken zu trainieren, um die genannten Vorteile für den Patienten zu erreichen [14]. OPCAB kann bei unsachgemäßer Anwendung die Komplikationsraten bekanntermaßen erhöhen und zu schlechteren Bypassqualitäten führen. Um dies, auch in der Lernphase, zu vermeiden, soll der Operationsablauf standardisiert sein, also klar definierten Schritten und festen Regeln folgen (z. B. RIVA-Anastomose immer zuerst ohne Manipulationen des Herzens, Benutzung des intrakoronaren Shunt, Kombination der Kompressenschlinge mit dem Herz-Positionierer, Kommunikationsregeln mit der Anästhesie). Dies führt zu Sicherheit sowie Kontrollierbarkeit und vermeidet Stress für den Chirurgen. Unter den gegebenen Trainingsmodalitäten ist ein längeres, expertenbegleitetes Training zu bevorzugen.

\section{Literatur}

1. Khan NE, De Souza A, Mister R, Flather M, Clague J, Davies S, Collins P, Wang D, Sigwart U, Pepper J (2004). A randomized comparison of off-pump and on-pump multivessel coronary-artery bypass surgery. N Engl J Med. 350(1):21-28

2. Magee MJ, Alexander JH, Hafley G, Ferguson TB Jr, Gibson CM, Harrington RA, Peterson ED, Califf RM, Kouchoukos NT, Herbert MA, Mack MJ (2008) PREVENT IV Investigators.Coronary artery bypass graft failure after on-pump and off-pump coronary artery bypass: findings from PREVENT IV. Ann Thorac Surg. Feb;85(2):494-499

3. Puskas, J; Cheng, D; Knight, J; Angelini, G; DeCannier, D; Diegeler, A; Dullum, M; Martin, J; Ochi, M; Patel, N; Sim, E; Trehan, N; Zamvar, V (2005) OffPump versus Conventional Coronary Artery Bypass Grafting: A Meta-Analysis and Consensus Statement From The 2004 ISMICS Consensus Conference. Innovations: Technology \& Techniques in Cardiothoracic \& Vascular Surgery. 1(1):3-27

\section{Korrespondierender Autor

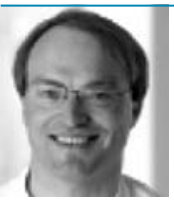 \\ Dr. med. Alexander Albert \\ Klinik für Herz-Thorax- und Gefäßchirurgie \\ Herzzentrum Lahr/Baden \\ Hohbergweg 2, 77933 Lahr, BRD \\ E-Mail: alexander.albert@heart-lahr.com}

Dr. med. Alexander Albert, Jahrgang 1966, absolvierte das Studium der Humanmedizin in Aachen und Freiburg. Er promovierte im Fach Neurologie an der Universität Basel. Von 1995 bis 2002 durchlief Albert die Ausbildung zum „Facharzt für Herzchirurgie“ in Lahr und Basel und ist seit 2000 Oberarzt im Herzzentrum Lahr. Schwerpunkte seiner Arbeit sind OPCAB und TAR, gerüstlose Bioklappen sowie Datamining.

Interessenkonflikt. Der korrespondierende Autor gibt an, dass kein Interessenkonflikt besteht. 
4. Peck E, Sergeant P (2007) Off-Pump Coronary Artery Bypass Graft Surgery. In: Yuh DD, Vricella LA, Baumgartner WA (Hrsg) The Johns Hopkins Manual of Cardiothoracic Surgery. New York, New York, McGraw Hill, S449-467

5. Albert A, Peck EA, Wouters P, Van Hemelrijck J, Bert C, Sergeant $P$ (2006) Performance analysis of interactive multimodal CME retraining on attitude toward and application of OPCAB. J Thorac Cardiovasc Surg 131(1):154-162

6. Wanzel KR, Ward M., Reznick RK (2002) Teaching the surgical craft: from selection to certification. Curr Probl Surg:583-659

7. Mack MJ (2004) Off-Pump Coronary Artery Bypass Graft Surgery. In: Yang SC, Cameron DE (Hrsg) Current Therapy in Thoracic and Cardiovascular Surgery. Mosby, Inc., An Affiliate of Elsevier, Philadelphia, Pennsylvania, S658-662

8. Khonsari S, Sintek C (2007) Cardiac Surgery: Safeguards and Pitfalls in Operative Technique. Lippincott Williams \& Wilkins Publishers; 4nd edition S 169-173

9. Song HK, Puskas JD (2006) In: Kaiser LR, Kron IL, Spray TL (eds.) Mastery of Cardiothoracic Surgery. 2nd Edition, Philadelphia, Pennsylvania, Lippincott Williams \& Wilkins, pp 454-465

10. Légaré Jean-François, Buth Karen J, Hirsch Gregory $\mathrm{M}$ (2005) Conversion to on pump from OPCAB is associated with increased mortality: results from a randomized controlled trial, Eur J Cardiothorac Surg; 27:296-301

11. Jenkins D, Al Ruzzeh S, Khan S, Bustami M, Modine T, Yacoub M, et al. (2002) Multi-vessel off-pump coronary artery bypass grafting can be taught to trainee surgeons. Heart Surg Forum; 5 Suppl 4: S342-S354

12. Raja SG (2005) Pump or no pump for coronary artery bypass: current best available evidence.Tex Heart Inst J; 32(4):489-501 (Review)

13. Sellke FW, DiMaio JM, Caplan LR, et al. (2005) American Heart Association. Comparing on-pump and off-pump coronary artery bypass grafting: numerous studies but few conclusions: a scientific statement from the American Heart Association council on cardiovascular surgery and anesthesia in collaboration with the interdisciplinary working group on quality of care and outcomes research. Circulation. May 31;111(21):2858-2864

14. Sergeant $P$, Wouters $P$, Meyns B, Bert C, Van Hemelrijck J, Bogaerts $C$, et al. (2004) OPCAB versus early mortality and morbidity: an issue between clinical relevance and statistical significance. Eur J Cardiothorac Surg; 25:779-785

\section{R. Larsen \\ Anästhesie und Intensivmedizin für die Fachpflege}

Berlin Heidelberg New York: Springer 2007.

7., vollst. überarb. Aufl., 1212 S., 416 Abb.

(ISBN 978-3-540-72273-1), geb., 44.00 EUR

Es ist wohl ein Kopf-an-Kopf-Rennen: die Auflagen der beiden Ein-Mann-Bücher von Larsen, nämlich, der Larsen, Anästhesie, 8. Auflage (für die Ärzte) und der Larsen, Anästhesie und Intensivmedizin für die Fachpflege, 7. Auflage. Allein diese hohen Auflagen lassen den Schluss zu, beide Bücher als Standardwerke des Faches zu bezeichnen. Denn beide erfreuen sich bei den avisierten Zielgruppen größter Beliebtheit - und nicht nur dort. Auch Medizinstudenten einschl. der PJ-Studenten nehmen diese Bücher entweder als Einstiegspforte oder als Vertiefung im Fach gern zur Hand. Ja selbst anästhesiologische Sachverständige benutzen längst zumindest den Larsen, Anästhesie als Messlatte zum von der Justiz gewünschten Aufzeigen der Differenzen zwischen dem medizinischen Standard und dem tatsächlichen zu beurteilenden Geschehen.

Die 7. Auflage des Lehrbuchs für die Fachpflege ist nun wiederum vom Autor vollständig überarbeitet worden. Geblieben sind erfreulicherweise der so geschätzte flüssige Schreibstil, die zahlreichen farbigen Abbildungen und Tabellen sowie die einprägsamen Merksätze. Alles zusammen lässt die Lektüre des Buches wieder zu einem Lesevergnügen werden, bei dem so ganz nebenbei natürlich auch und wesentlich Wissen vermittelt wird.

Neu sind hingegen am Ende eines jeden Kapitels die Literaturhinweise und Internetadressen, die Wissbegierige in der Thematik weiterführen können. Zusammen mit den vom Autor formulierten Fragen zum Selbsttest fordern sie den Leser auf zur kritischen Überprüfung des soeben gelernten Stoffes. Das ist geradezu eine ideale Vorbereitung auf entsprechende Prüfungen in der Fachpflege, ist aber bestimmt auch für Staatsexamina der Medizinstudenten gut geeignet.

Eine stattliche Anzahl der insgesamt 72 Kapitel sind vollkommen neu konzipiert worden und gehen inhaltlich z. T. weit über den sonst üblichen Rahmen von anästhesiologischen Lehrbüchern für die Fachpflege hinaus; einige Teile von Kapiteln überschrei- ten aber wohl auch schon das vom Fachpflegepersonal gewünschte Wissen (z. B. „Narkose beim Coiling von Hirnaneurysmen"). Man hat den Eindruck, dass hier fast schon eine gewollte (?) inhaltliche Annäherung beider Lehrbücher durch Larsen geschieht. Vielleicht sollte Larsen doch stets bedenken, für welche Zielgruppe er gerade die nächste Neuauflage konzipiert.

Nichtsdestoweniger: Auch die 7. Auflage der Anästhesie und Intensivmedizin für die Fachpflege ist wieder äußerst empfehlenswert.

J. Radke (Halle/Saale) 